\title{
Artificial Language Learning in Adults and Children
}

\author{
Vasiliki Folia \\ Max Planck Institute for Psycholinguistics \\ Radboud University Nijmegen \\ Stockholm Brain Institute
}

Julia Uddén

Max Planck Institute for Psycholinguistics

Radboud University Nijmegen

Stockholm Brain Institute

Meinou de Vries

Max Planck Institute for Psycholinguistics

\section{Christian Forkstam}

Max Planck Institute for Psycholinguistics

Radboud University Nijmegen

Stockholm Brain Institute

Universidade do Algarve

\section{Karl Magnus Petersson}

Max Planck Institute for Psycholinguistics

Radboud University Nijmegen

Stockholm Brain Institute

Universidade do Algarve

\footnotetext{
This work was supported by the Max Planck Institute for Psycholinguistics, Donders Institute for Brain, Cognition and Behaviour, FCT grant IBB/CBME, LA, FEDER/POCI 2010, Vetenskapsrådet (8276), Hedlunds Stiftelse, and Stockholm County Council (ALF, FoUU).

Correspondence concerning this article should be addressed to Karl Magnus Petersson, Max Planck Institute for Psycholinguistics, P.O. Box 310, NL-6500 AH Nijmegen, the Netherlands. Internet: karl-magnus.petersson@mpi.nl
} 
This article briefly reviews some recent work on artificial language learning in children and adults. The final part of the article is devoted to a theoretical formulation of the language learning problem from a mechanistic neurobiological viewpoint and we show that it is logically possible to combine the notion of innate language constraints with, for example, the notion of domain general learning mechanisms. A growing body of empirical evidence suggests that the mechanisms involved in artificial language learning and in structured sequence processing are shared with those of natural language acquisition and natural language processing. Finally, by theoretically analyzing a formal learning model, we highlight Fodor's insight that it is logically possible to combine innate, domain-specific constraints with domain-general learning mechanisms.

Human languages are characterized by the "design features of language" (Hockett, 1963, 1987): discreteness, arbitrariness, productivity, and the duality of patterning (i.e., elements at one level are combined to construct elements at another). Somehow these properties arise from how the human brain works, develops, and learns in interaction with its environment. For example, a study characterizing the emergence of Nicaraguan Sign Language (NSL) (Senghas, Kita, \& Özyürek, 2004) showed how two of Hockett's design features - segmentation/discretization and combinatoriality-rapidly emerged in the population of NSL signers. Similarly, Aronoff, Meir, Padden, and Sandler (2008) documented the development of morphology and syntax in Al-Sayyid Bedouin signers and described the emergence of recursive syntax from the first generation onward (see also Sandler, Meir, Padden, \& Aronoff, 2005). One way to interpret these findings is that humans are equipped with learning mechanisms that shape the language acquired into discrete and hierarchically organized system when the relevant communicative context is present.

The human capacity for language and communication is subserved by an intricate network of brain regions that collectively instantiate the semantic, syntactic, phonological, and pragmatic operations necessary for adequate comprehension and production. How are these skills acquired? Despite much progress, it is still not well understood how humans acquire language skills. The acquisition of language is a complex learning task that is governed by constraints deriving from the properties of the developing human brain. These constraints, to the extent that they are innate (genetic or nongenetic), need not be acquired. The mainstream generative position has for a long time been that there are interesting language acquisition constraints that are linguistic in nature and that language is acquired by means of a language-specific acquisition device (Chomsky, 1965, 1986; Jackendoff, 2002). During the last decade, this position 
has become increasingly challenged on processing grounds (e.g., Christiansen \& Chater, 1999; Reali \& Christiansen, 2009), on evolutionary and acquisition grounds (e.g., Chater \& Christiansen, 2009; Christiansen \& Chater, 2008; Pullum \& Scholz, 2002; Scholz \& Pullum, 2002), as well as on grounds of language diversity (Evans \& Levinson, 2009). The alternative proposal suggests that children make use of domain-general learning mechanisms (e.g., Chater \& Christiansen, 2009; Christiansen \& Chater, 2008). However, this latter position is not incompatible with the notion that language acquisition has an innate basis (Chater \& Christiansen, 2009; Chater, Reali, \& Christiansen, 2009). Rather, it is suggested that this basis in large part is non-language-specific, or prelinguistic, in nature (Hornstein, 2009).

During the past decade, artificial language learning (ALL) paradigms have revitalized the study of language acquisition and language evolution (e.g., Bahlmann, Schubotz, \& Friederici, 2008; Christiansen \& Chater, 2008; Christiansen \& Kirby, 2003; de Vries, Monaghan, Knecht, \& Zwitserlood, 2008; Fitch \& Hauser, 2004; Forkstam, Hagoort, Fernandez, Ingvar, \& Petersson, 2006; Forkstam, Jansson, Ingvar, \& Petersson, 2009; Friederici, Bahlmann, Heim, Schubotz, \& Anwander, 2006; Friederici, Fiebach, Schlesewsky, Bornkessel, \& von Cramon, 2006; Friederici, Steinhauer, \& Pfeifer, 2002; Gentner, Fenn, Margoliash, \& Nusbaum, 2006; Gómez \& Gerken, 1999, 2000; Hauser, Chomsky, \& Fitch, 2002; Makuuchi, Bahlmann, Anwander, \& Friederici, 2009; Marcus, Vijayan, Bandi Rao, \& Vishton, 1999; Misyak, Christiansen, \& Tomblin, 2009; Perruchet \& Rey, 2005; Petersson, Forkstam, \& Ingvar, 2004; Poletiek, 2002; Saffran, Aslin, \& Newport, 1996; Uddén et al., 2009). The complexity of natural languages makes it exceedingly difficult to isolate factors responsible for language learning. For example, in natural language processing, semantics, syntax, and phonology operate in parallel, in close spatial and temporal contiguity, and because of this, artificial language learning paradigms have been developed with the objective of controlling the influence of the various elements of natural language. Language researchers have thus turned to artificial languages as a means of obtaining better experimental control over the input to which learners are exposed. For example, the use of artificial languages makes it possible to control for prior learning. Moreover, it is crucial to know what children can learn in order to specify possible language acquisition mechanisms. More importantly, the identification of such learning mechanisms will allow researchers to evaluate their degree of domain-specificity as well as possible inherent constraints. The basic assumption in artificial language learning research is that some of the learning mechanisms are shared between artificial and natural language acquisition (Gómez \& 
Gerken, 2000; Petersson et al., 2004; Reber, 1967). In addition, artificial grammar learning (AGL) experiments, a version of ALL experiments that focuses on syntax (i.e., sequential structure), has been used in cross-species comparisons to establish which, if any, are the uniquely human components or properties of the language faculty (Fitch \& Hauser, 2004; Gentner et al., 2006; Hauser, Chomsky et al., 2002; Newport \& Aslin, 2004; Newport, Hauser, Spaepen, \& Aslin, 2004; O’Donnell, Hauser, \& Fitch, 2005; Saffran et al., 2008).

\section{Artificial Syntax Learning in Children}

The current lack of knowledge concerning the actual learning mechanisms involved during infancy makes it difficult to determine the relative contributions of innate and acquired knowledge in language acquisition. One approach to these issues exposes infants to artificial languages and this approach has resulted in a number of discoveries regarding the learning mechanisms available during infancy (Gómez \& Gerken, 2000).

The difficulty of acquiring a language is related to the fact that the internal mental structures that represent linguistic information are not directly expressed in the surface form of a language (e.g., the utterance). The question of if and how these structures are acquired is the question of how a learner transforms the language input ("primary linguistic data") into phonological, syntactic, and semantic knowledge (Chomsky, 1980b). Under the traditional Chomskyan view, the input underdetermines the linguistic knowledge of the adult grammar. The dilemma of generalizing beyond the stimuli encountered without overgeneralizing, in combination with the absence of certain generalization errors during child language acquisition, suggests that the learning mechanisms involved are constrained by prior knowledge or constraints. For example, it appears that children never consider rules solely based on linear order in sentences (Gómez \& Gerken, 2000). This and similar observations was one of the fundamental reasons that led Chomsky to propose the existence of a specific language acquisition device (Chomsky, 1965, 1986, 2005). Thus, the acquisition of a grammar is not only based on an analysis of the linguistic input but also depends on an innate structure that guides the process of language acquisition (Jackendoff, 2002).

Recently, Lidz, Waxman, and Freedman (2003) investigated the syntactic structures required to determine the antecedent for the pronoun "one." Based on corpus analyses of child-directed speech in the CHILDES database (MacWhinney, 2000), they concluded that the anaphoric uses of "one" that are syntactically uninformative vastly outstrip the informative use in the input. 
Lidz et al. (2003) reported that the data that the infant would need in order to learn the syntax of "one" occur at a rate that is indistinguishable from noise in the input. Interestingly, Lidz and colleagues (2003) also showed that 18-month-old infants have acquired the syntax of "one" and concluded that this constitutes evidence for a contribution of innate structure (for a different view, see Chater \& Christiansen, 2007). Similarly, longitudinal data on Korean reveal that positive evidence for multiple nominative cases and scrambling is extremely rare, presenting a poverty-of-the-stimulus situation (Kang, 2008). Kang's study (2008) provides evidence that children do match input frequency by statistical learning abilities but argues that this cannot explain how children acquire language-specific properties under situations of impoverished input. Instead, Kang suggested that in order to acquire these syntactic structures, children must use prior knowledge specific to language, supporting the tenet of the principles and parameters theory of language acquisition (Chomsky \& Lasnik, 1995).

Artificial language research in children has focused on four aspects of the language learner's task (Gómez \& Gerken, 2000): (a) segmentation; (b) acquisition of sequence regularities; (c) generalization of structural (i.e., syntactic) relations; and (d) acquisition of syntactic categories. We note here that the artificial language paradigms that have been investigated in both adults and children generally report similar or "equivalent" findings for both adults and children, although interesting differences have also been reported.

\section{Word Segmentation}

An important acquisition task for infant learners is to identify word boundaries in continuous speech. Results from several artificial speech paradigms have shown that infants are equipped with learning mechanism(s) allowing them to become sensitive to transitional probabilities over artificial syllable sequences (Saffran et al., 1996). The evidence for these learning mechanism(s) in infants are largely based on artificial language learning paradigms, in which children are typically exposed to continuous speech and the transition probabilities between syllables are manipulated. The infants are then tested on their ability to discriminate words and nonwords or parts of words, which they do better than chance (Aslin, Saffran, \& Newport, 1998). Similar findings have been reported for tone sequences (Saffran, Johnson, Aslin, \& Newport, 1999) and visual sequences (Kirkham, Slemmer, \& Johnson, 2002). In this context, Gómez and Gerken (2000) raised the question of whether children actually use the probabilistic sequence regularities for real word segmentation and treat the segments as lexical items. Recently, Pelucchi, Hay, and Saffran (2009) 
showed that English-learning 8-month-old children were able to track transitional probabilities in fluent infant-directed Italian speech and argued that their result supports the claim that statistical learning is sufficient to support aspects of actual language acquisition.

\section{Acquisition of Structured Sequence Knowledge}

The complement to segmentation is sequencing. Thus, the language learner must acquire structured sequence regularities, including word order regularities, in addition to words during language acquisition. In this section we review some results on pattern-based abstraction and category-based generalization in the acquisition of sequence structure, but first we provide a brief summary of earlier results. In an early study, Gómez and Gerken (1999) showed that after brief exposure to a simple artificial grammar, 12-month-old children could distinguish new grammatical from nongrammatical sequences, suggesting that learners were able to generalize the acquired knowledge to new sequences with familiar co-occurrence patterns. Gómez and Gerken (1999) also showed that children were able to discriminate grammatical and nongrammatical sequences in a transfer version of their artificial language learning paradigm (i.e., despite the change of vocabulary). Gómez and Gerken (2000) argued that their findings suggest that the infant brain supports abstraction processes for the acquisition of syntactic structure, consistent with the infant capacity for rapid rule abstraction (Marcus et al., 1999). Subsequently, Marcus, Johnson, Fernandes, and Slemmer (2004) showed that infants might not be able to do this for certain types of nonlinguistic stimuli (e.g., tones and shapes), and they suggested that this type of rule abstraction therefore is specific to language. However, it was recently shown that infants can acquire these rules with familiar, salient nonlinguistic material, like familiar animals (Saffran, Pollak, Seibel, \& Shkolnik, 2007). Interestingly, cotton-top tamarin monkeys were also able to acquire the rules used by Marcus et al. (1999) using linguistic stimuli (Hauser, Weiss, \& Marcus, 2002).

Pattern-based abstraction can be described in terms of relations over surface-based (e.g., physical) characteristics of the stimuli. A relation is abstracted by comparing the perceptual characteristics of elements in a sequence (Gómez \& Gerken, 2000). Such structures, or patterns of relations, are surfacebased abstractions and infants are sensitive to such pattern-based abstraction (Gómez \& Gerken, 1999; Marcus et al., 1999). Recently, Saffran et al. (2007) provided evidence suggesting that this type of rule learning is not domainspecific (i.e., limited to linguistic stimuli), but also holds for nonlinguistic 
material (e.g., sequences of dog pictures). Consistent with this, cotton-top tamarins also master this type of rule learning (Hauser, Weiss et al., 2002).

These rule-learning tasks are more than just simple sequence learning. The learner must, for example, detect the same/difference relationships within sequences, requiring the learner to represent and categorize sequence tokens as being of the same or different type. Thus, factors such as stimulus familiarity, categorizability, and ease of representation, are important factors that might modulate acquisition (Saffran et al., 2007). Saffran et al. (2007) suggested, more generally, that pattern learning is facilitated when the perceptual information presented match the relevant learning mechanism and, in this sense, learning mechanisms are constrained by the nature of the information to be acquired. Gerken (2006) provides interesting results in this context, replicating the findings of Marcus et al. (1999) in two experiments in which infant learners were exposed to different acquisition sets, generated from the same artificial grammar and with several plausible generalizations possible. The results showed that one group of learners generalized in one direction, whereas the other did not, and this depended on the acquisition set. Gerken (2006) suggested that learners behave conservatively and do not generalize too far beyond the regularities present in the input. This also suggests that the structure of the acquisition set, or stimulus domain, influences the type of regularities that the learner will be tracking (Gerken, 2006). Similarly, the results of Saffran et al. (2005) suggest that the structure of the input determines the primitives over which generalizations are made, which presumably are part of an innate endowment, previously acquired, or both. This type of research, in which the generalization properties of the acquisition machinery is characterized as a function of the input data is of crucial importance to the potential to distinguish between theories of language development.

In contrast to pattern-based abstraction, category-based generalization involves operations over abstract rather than perceptually bound variables. Gómez and Gerken (2000) illustrated this point by comparing the pattern-based representation ABA with the category-based representation Noun-Verb-Noun. Recognizing ABA and Noun-Verb-Noun both involve identity. In the ABA case, the relation is surface bound and related to the identity of two tokens of the same type $(A=A)$, whereas in the Noun-Verb-Noun case, the identity relation holds over categories (Noun $=$ Noun). In the latter case, the learner has to identify the first and third elements as members of the category Noun.

The ability to abstract over categories is fundamental to natural language acquisition/processing. One hypothesis is that a learner who identifies a novel word as belonging to a particular category has immediate access to all of 
the rules involving that category. Category-based abstraction and the problem of how learners acquire relations between grammatical classes are therefore central to understanding language acquisition. Whereas abstract arbitrary dependencies in general are difficult to acquire, if a subset of category members are conceptually or perceptually marked, the acquisition task might become easier - abstraction seem to occur when there is sufficient evidence to distinguish the categories (Gómez \& Gerken, 2000). This suggests constraints on the learner and the nature of the acquisition mechanisms (Braine, 1987).

In a recent study, Gerken, Wilson, and Lewis (2005) investigated whether syntactic category formation in children based on distributional properties alone is possible. In this study, American children (18 months), briefly familiarized with a partial Russian gender paradigm, discriminated between new grammatical and ungrammatical items, but like adults in previous studies, they were only able to do so when a subset of the familiarization items was doubly marked for gender category. Gerken et al. argued that learners are able to use distributional cues to detect category structure without referential cues from relatively early on in the language learning process. One important issue that is raised by these findings is how the learning system knows which cues are relevant in a given context. In general, almost any stimulus material contains a multitude of cue dimensions and one central issue is therefore to identify the source(s) of cue selectivity.

Finally, there is one important domain - the mapping out of developmental trajectories under experimental control — in which infant research on artificial language acquisition cannot be replaced by corresponding adult research. An interesting example was recently reported by Gómez and Maye (2005), who investigated the acquisition of simple nonadjacent dependencies in infants. The results suggested that whereas 15 -month-old children were able to acquire sensitivity to a simple nonadjacent dependency structure, this was not the case for 12-month-olds. This developmental dissociation might be understood in terms of, for example, differences in the size of online processing window, modulated by attention and working memory capacities, differences in representational capacities, or innate developing biases - all topics for future research.

A crucial assumption in research on artificial language learning and structured sequence processing is, of course, that the mechanisms involved are shared with natural language acquisition and processing. A growing body of evidence suggests that this is indeed the case. This includes evidence from studies using functional magnetic resonance imaging (fMRI) (Forkstam et al., 2006; Petersson, Folia, \& Hagoort, 2010; Petersson et al., 2004), electroencephalography (EEG) (Friederici et al., 2002; Hoen \& Dominey, 2000), and 
transcranial magnetic stimulation (TMS) (Uddén et al., 2008; Uddén, Ingvar, Hagoort, \& Petersson, 2010a). Furthermore, behavioral investigations also suggest that artificial language learning/processing is relevant to natural language learning/processing, including parallel developmental trajectories mapped with artificial (Gómez \& Maye, 2005) and natural language material (Santelmann \& Jusczyk, 1998), as well as brain lesion studies suggesting that language processing deficits are paralleled by impairment in structured sequence learning/processing (Christiansen, Kelly, Shillcock, \& Greenfield, 2010; Evans, Saffran, \& Robe-Torres, 2009; Hoen et al., 2003; Hsu, Christiansen, Tomblin, Zhang, \& Gómez, 2006; Reali \& Christiansen, 2009; Richardson, Harris, Plante, \& Gerken, 2006).

\section{Artificial Syntax Learning in Adults}

The acquisition of sequence structure knowledge is typically investigated in various artificial grammar learning (AGL) paradigms. The implicit AGL paradigm provides one approach to systematically investigating aspects of structural (i.e., syntactic) acquisition from exposure to grammatical (i.e., positive) examples alone, without explicit feedback, teaching instruction, or engaging subjects in explicit problem solving by instruction (Petersson et al., 2004). In certain important respects, these acquisition conditions resemble those found in natural language development (Reber, 1967). Generally, AGL consists of acquisition and test phases. In the acquisition phase, participants are exposed to an acquisition set generated from a formal grammar. In the standard version, subjects are informed after acquisition that the sequences were generated according to a complex set of rules (but they are not told about the actual rules), and they are asked to classify novel sequences as grammatical or not, based on their immediate "gut feeling." A robust finding is that subjects classify well above chance, both for regular (e.g., Folia et al., 2008; Forkstam, Elwér, Ingvar, \& Petersson, 2008; Petersson et al., 2004; Stadler \& Frensch, 1998) and nonregular dependencies, including context-sensitive nonadjacent dependencies (Uddén et al., 2009).

\section{Behavioral Findings: Regular and Nonregular Dependencies}

An alternative way to assess implicit artificial syntax acquisition is the structural mere exposure version of AGL, in which participants are never informed about an underlying generative mechanism (Folia et al., 2008; Forkstam et al., 2008). This version is based on the "mere exposure effect," which refers to the finding 
that repeated exposure to a stimulus induces an increased preference for that stimulus compared to novel stimuli (Zajonc, 1968).

In structural mere exposure AGL, participants are asked to make preference judgments on novel (like/prefer or not) sequences, based on their immediate intuitive impression. Folia et al. (2008) investigated both grammaticality and preference classification after 5 days of implicit acquisition on sequences generated from a simple right-linear unification grammar (cf., e.g., Petersson et al., 2010). The grammaticality task was only administered after the last preference classification on the last day of the experiment. The results showed that the participants performed well above chance on both preference and grammaticality classification (Figure 1). Participants improved their performance on grammaticality compared to preference classification. However, this difference was quantitative rather than qualitative in nature, as all effects significant in grammaticality classification were already significant in preference classification, and the reverse; only the pattern of results was strengthened in grammaticality compared to preference classification. In addition to the factor grammaticality status, we also manipulated a measure of local subsequence familiarity - associative chunk strength (high/low ACS, cf., Folia et al., 2008; Forkstam et al., 2006; Knowlton \& Squire, 1996; Meulemans \& Van der Linden, 1997). The effect of local subsequence familiarity on endorsement rates was only modest compared to the actual grammaticality status (Figure 1). There was no significant interaction between grammaticality status and local subsequence familiarity (ACS). Thus, these results suggest that structural knowledge independent of ACS is used to classify novel sequences and provides support for the notion that syntactic structure, other than local subsequence regularities, is used for classification. Subjective reports also showed that the participants did not utilize rule searching or other explicit problem-solving strategies but that their classification decisions were reached by guessing based on "gut feeling." Moreover, the subjective ratings of perceived performance did not correlate with the actual performance. Very similar results were also found in another preference/grammaticality study of adult learners (Forkstam et al., 2008).

Uddén and colleagues (Uddén et al., 2009; Uddén, Ingvar, Hagoort, \& Petersson, 2010b) investigated implicit acquisition of nested and crossed nonadjacent dependencies (corresponding to context-free and context-sensitive grammars, respectively) while controlling for local subsequence familiarity. In contrast to many AGL studies, we used an implicit learning paradigm over 9 days. This provides enough time for both abstraction processes and knowledge consolidation to take place. This is important in implicit AGL because sleep has a significant effect on classification performance in adults (Nieuwenhuis, 


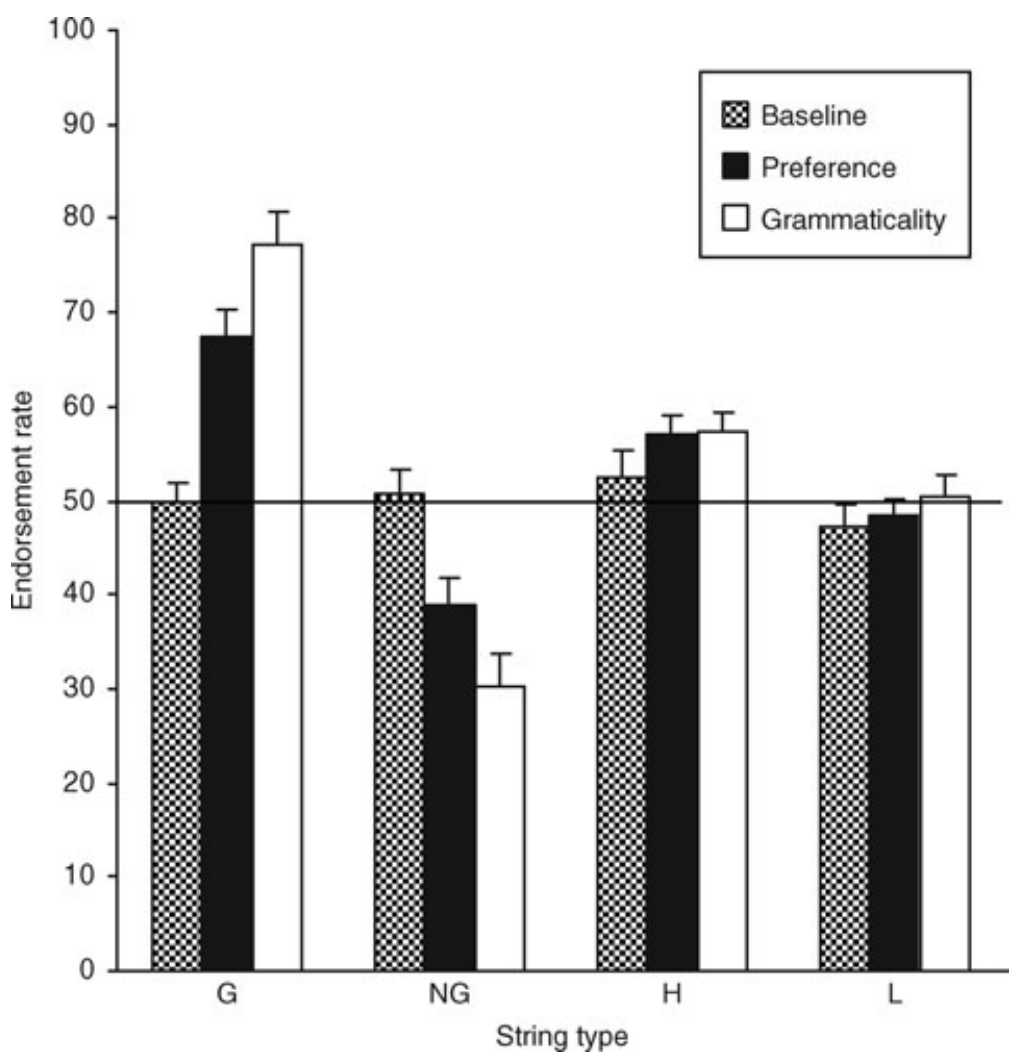

Figure 1 Endorsement rates over grammaticality and ACS main factor categories. The endorsement rates (i.e., items classified as grammatical independent of actual grammaticality status $)$ as a function of grammaticality status $(\mathrm{G}=$ grammatical sequences, $\mathrm{NG}=$ nongrammatical sequences $)$ as well as associative chunk strength $(\mathrm{H}=$ high ACS sequences, $\mathrm{L}=$ low ACS sequences). Error bars correspond to standard error of the mean.

Folia, Forkstam, Jensen, \& Petersson, 2010). This is consistent with results that naps promote abstraction processes after artificial language learning in 15-month-old infants (Gómez, Bootzin, \& Nadel, 2006).

In the first experiment, Uddén and colleagues (Uddén et al., 2009; Uddén et al., 2010b) directly compared the acquisition of regular and nonregular syntax (i.e., nested dependencies) in a within-subject design. We found that subjects implicitly acquired knowledge about the nonadjacent nested dependencies. However, the acquisition of nonregular aspects was harder than regular 


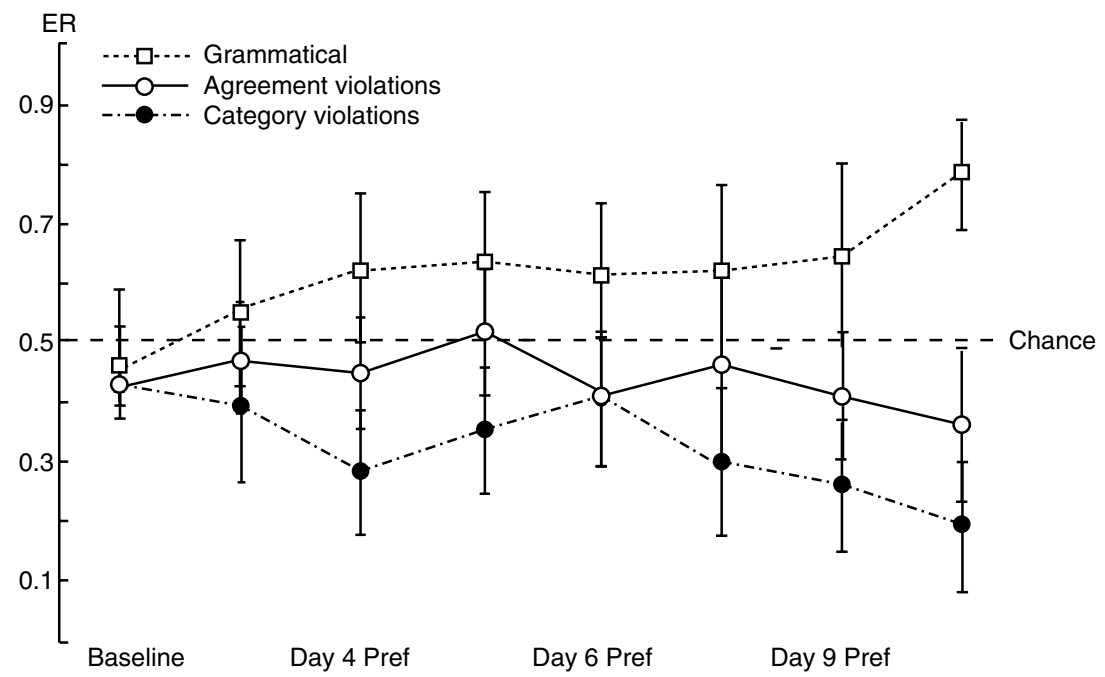

Day 3 Pref Day 5 Pref Day 7 Pref Day 9 Gram

Figure 2 Classification performance in endorsement rates over the nine days of the experiment. Pref $=$ preference classification, which was also used in the baseline test; Gram $=$ grammaticality classification. Squares are grammatical sequences, empty circles are agreement violation sequences, and filled circles are category violation sequences. Error bars indicate the standard deviation.

aspects of the underlying grammar. In the second experiment, we investigated an agreement structure that generated context-sensitive, crossed dependencies. The nongrammatical sequences consisted of two violation types: category violations $\left(\mathrm{A}_{1} \mathrm{~A}_{2} \mathrm{~A}_{3} \mathrm{~A}_{4} \mathrm{~B}_{2} \mathrm{~B}_{1}\right)$ and agreement violations (e.g., $\left.\mathrm{A}_{1} \mathrm{~A}_{3} \mathrm{~A}_{2} \mathrm{~B}_{3} \mathrm{~B}_{2} \mathrm{~B}_{1}\right)$. The results replicated the finding that participants implicitly acquire nonregular structure. In addition, the results showed that agreement violations were significantly harder to reject than category violations (Figure 2).

In the third experiment, we employed a between-subject design to compare the implicit acquisition of context-sensitive, crossed dependencies (e.g., $\left.\mathrm{A}_{1} \mathrm{~A}_{2} \mathrm{~A}_{3} \mathrm{~B}_{1} \mathrm{~B}_{2} \mathrm{~B}_{3}\right)$ and the more commonly studied context-free, nested dependencies (e.g., $A_{1} A_{2} A_{3} B_{3} B_{2} B_{1}$ ). The results showed robust classification performance, equivalent to levels observed with regular grammars, for both types of nonregular dependencies (Figure 3). The postexperimental questionnaire showed that although participants accepted that there were correspondences between symbols in the sequences significantly more often than chance, when they were provided with the correct agreement constraints they performed at 

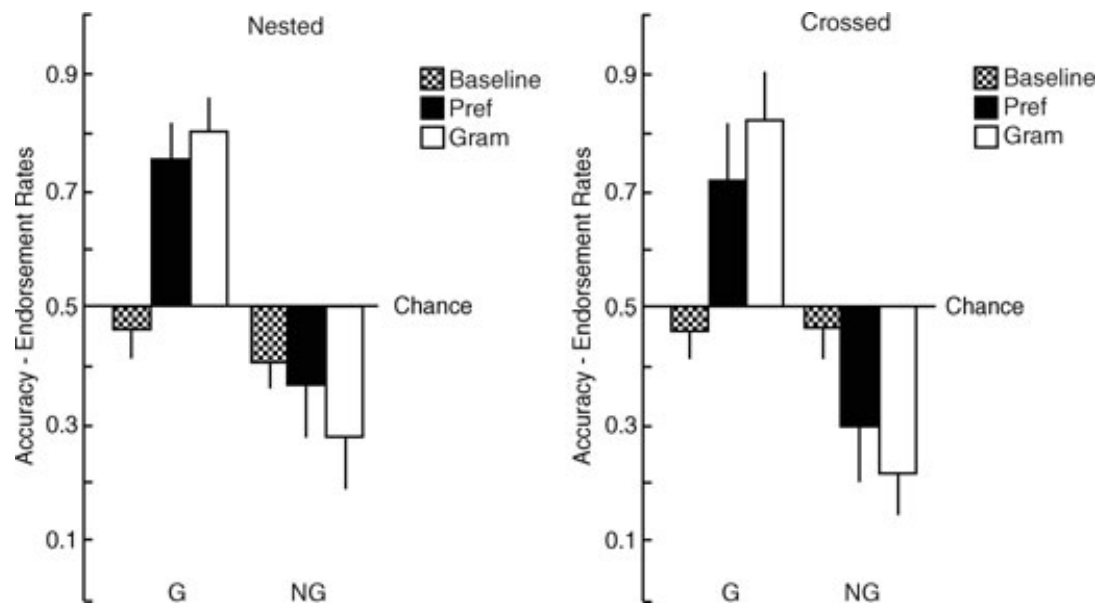

Figure 3 Classification performance in endorsement rates. Pref $=$ preference classification, which was also in the baseline test; Gram = grammaticality classification. Error bars indicate standard deviations.

chance level in terms of accepting or rejecting them. Thus, as in the previous experiments, there was little evidence for any explicit knowledge of the underlying grammar, supporting the notion that structural knowledge was implicitly acquired.

Similar findings have also been reported by others (de Vries, Monaghan, Knecht, \& Zwitserlood, 2010). In particular, de Vries et al. 2010 showed that learning of nonadjacent dependencies can be facilitated by perceptual cues that make the nonadjacent dependencies more salient. Taken together, these results show that preference and grammaticality classification are equivalent in terms of behavioral effects and strongly support the notion that humans can implicitly acquire knowledge about complex systems of interacting rules by mere exposure to the acquisition material. Moreover, the results show that if given enough acquisition exposure, participants demonstrated robust implicit learning of nonadjacent dependencies of context-free and context-sensitive types at levels comparable to simple right-linear structures.

\section{fMRI Findings}

In a recent fMRI study (Petersson et al., 2010), we investigated a simple right-linear unification grammar (cf., Hagoort, 2005; Joshi \& Schabes, 1997; Vosse \& Kempen, 2000). In an implicit AGL paradigm, subjects were exposed to grammatical sequences in an immediate short-term memory task and 


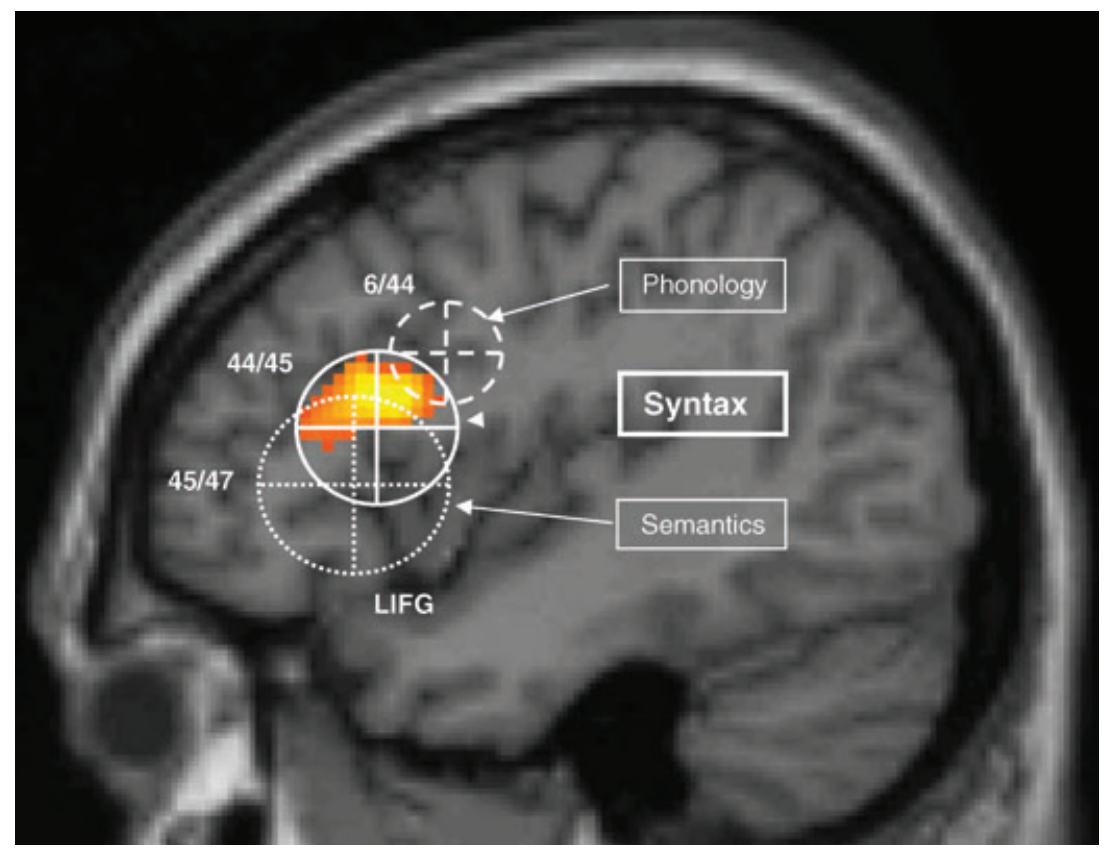

Figure 4 Regions related to phonological, syntactic, and semantic processing (cf., Hagoort, 2005). Left hemisphere: Activation related to artificial syntactic violations.

no performance feedback was provided. Implicit acquisition took place over 5 days. On the last day a grammaticality classification test was administered with the factors grammaticality and local subsequence familiarity (technically, ACS, cf. Knowlton \& Squire, 1996; Meulemans \& Van der Linden, 1997). In addition, natural language data from a sentence comprehension experiment had been acquired in the same subjects in a factorial design with the factors syntax and semantics (for details, see Folia, Forkstam, Hagoort, \& Petersson, 2009). The main results of this study (Petersson et al., 2010) replicate previous findings (Figure 4) on implicit AGL in detail (Forkstam et al., 2006; Petersson et al., 2004). First, in contrast to claims that Broca's region, in the context of language processing, is specifically related to syntactic movement (Grodzinsky \& Santi, 2008; Santi \& Grodzinsky, 2007a, 2007b) or the processing of nested dependencies (Bahlmann et al., 2008; Friederici, Bahlmann et al., 2006; Makuuchi et al., 2009), we found the left inferior frontal region centered on BA 44 and 45 to be activated during the artificial syntax processing of a well-formed (grammatical) sequence independent of local subsequence 
familiarity. Second, Broca's region was engaged to a greater extent for artificial syntactic violations - that is, when the unification of structural pieces becomes more difficult or impossible (cf., Hagoort, 2005). We note that the unification operator in any unification grammar is an incremental and recursive process (see Petersson et al., 2010, for details). The effects related to artificial syntactic processing in Broca's region were essentially identical when we masked these with activity related to natural syntax processing in the same subjects (Folia et al., 2009). Thus, these results were also highly consistent with functional localization of natural language syntax in the left inferior frontal gyrus (Figure 4) (Bookheimer, 2002; Hagoort, 2005). Interestingly, the medial temporal lobe was deactivated during artificial syntactic processing, consistent with the view that implicit processing does not rely on declarative memory mechanisms that engage the medial temporal lobe memory system (Forkstam \& Petersson, 2005; Knowlton \& Squire, 1996; Seger, 1994). Based on these and previous findings, Petersson et al. (2010) concluded that the left inferior frontal region is a generic online sequence processor that unifies information from various sources in an incremental and recursive manner, independent of whether there are requirements for syntactic movement or processing of nested nonadjacent dependencies.

Given the findings of Folia et al. (2008) as well as Uddén and colleagues (Uddén et al., 2009; Uddén et al., 2010b) that grammaticality and preference classification are essentially equivalent at the behavioral level after implicit acquisition, we decided to investigate this issue with fMRI. Participants were exposed to a simple right-linear unification grammar in an implicit AGL paradigm during 5 days.

On day 1, fMRI data was acquired during a baseline preference task in which participants had to classify sequences as likable/preferable or not based on their immediate "gut-feeling." There were no significant effects of grammaticality status or local subsequence familiarity on day 1 , neither at the brain nor the behavioral level. On day 5, the participants classified new sequences as likable/preferable or not. In contrast to the baseline preference classification, the preferences of the subjects now correlated significantly with the grammaticality status of the sequences both at the brain and behavioral level (Figures 1 and 5).

\section{TMS Findings}

One way to test whether the neural correlates of artificial syntax processing observed with fMRI in Broca's region (BA 44/45) is causally related to classification performance is to test whether repeated transcranial magnetic stimulation 


\section{Preference Classification: NG > G}

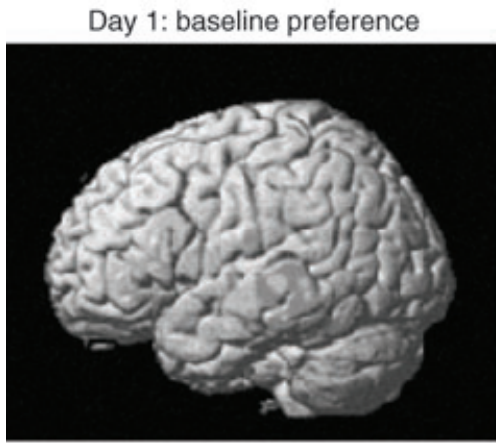

Day 5: preference classification

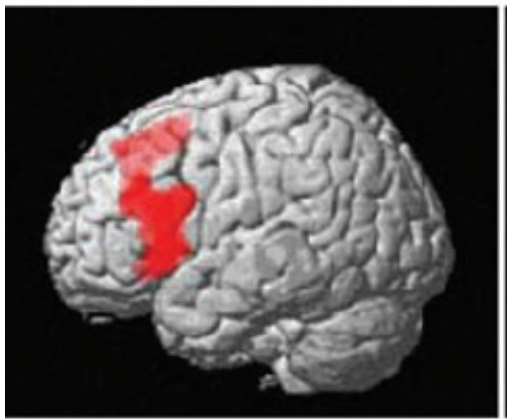

Implicit acquisition: D5 vs D1
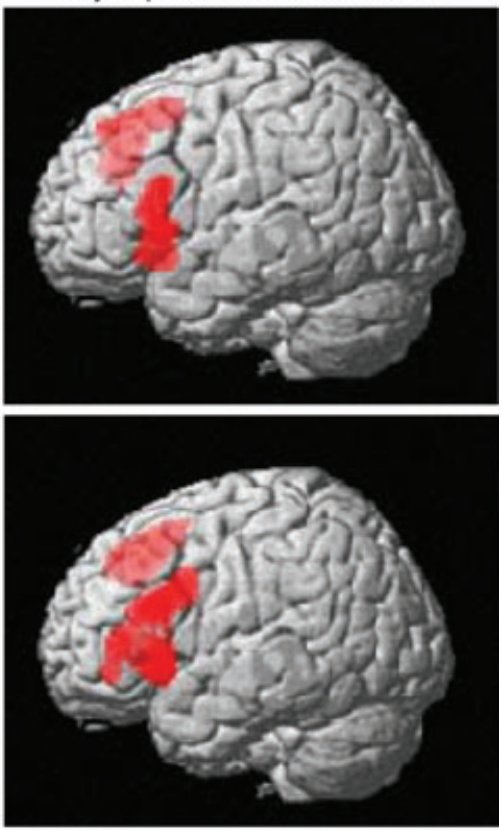

Overlap NLP and ALP

Figure 5 Brain regions engaged during correct preference classification. The main effect nongrammatical versus grammatical sequences on day 1 , baseline (preference) classification (top left); on day 5, and preference classification after 5 days of implicit acquisition (top right); the effect of implicit acquisition (lower left); and the main effect nongrammatical $(\mathrm{NG})>$ grammatical $(\mathrm{G})$ during grammaticality classification (lower right), here masked with the syntax related variability.

(rTMS) to Broca's region modulates classification performance. This approach has also been used to investigate natural language processing. The previous literature has reported both interference and facilitation effects on behavior after rTMS. For example, rTMS applied to the left prefrontal cortex improved analogical reasoning (Boroojerdi, Grafman, \& Cohen, 2001), whereas applied to Wernicke's region, language perception (Andoh et al., 2006) and picture naming were improved (Mottaghy, Sparing, \& Töpper, 2006; Töpper, Mottaghy, Brügmann, Noth, \& Huber, 1998). Findings of enhanced phonological memory, by eradication of the phonological similarity effect, after TMS of the left inferior parietal region has also been reported (Kirschen, Davis-Ratner, 
Jerde, Schraedley-Desmond, \& Desmond, 2006). These findings suggest that TMS perturbation of a neural network does not necessarily lead to impaired performance. On the other hand, there are also several studies of language processing in which TMS applied to the left prefrontal cortex produced impaired performance. Reaction time increases were observed in a production task with verbs but not nouns (Shapiro, Pascual-Leone, Mottaghy, Gangitano, \& Caramazza, 2001) and in a semantic but not a phonological control task (Devlin, Matthews, \& Rushworth, 2003). The latter dissociation was recently extended to a double dissociation between anterior and posterior left inferior frontal regions (Gough, Nobre, \& Devlin, 2005). In addition, impairments in the ability to read aloud (Epstein et al., 1999) and to match pictures to words (Flitman et al., 1998) have also been reported after TMS stimulation of the left Inferior Frontal Cortex (IFC). Taken together, these results suggest that the factors determining whether TMS will improve or degrade task performance are currently not well understood.

We have previously shown that Broca's region is causally involved in syntactic processing of sequences generated from a simple right-linear regular grammar (Uddén et al., 2008). In a recent follow-up (Uddén et al., 2010a), we investigated whether this was also the case for nonregular nonadjacent dependencies. More specifically, we investigated the same context-sensitive type of crossed dependencies as described earlier in an offline 1-Hz rTMS paradigm in which the left inferior frontal region (BA 44/45) and the vertex (control region) were stimulated. We also included non-TMS classification sessions, both preference and grammaticality classification, in order to replicate the previous behavioral findings. Participants implicitly acquired the crossed dependency structure and the TMS results showed that rTMS to Broca's region interferes with subsequent grammaticality classification of crossed nonadjacent dependencies. We found a significant performance decrease after Left Inferior Frontal Cortex (LIFC) stimulation compared to vertex stimulation (a control region irrelevant to syntactic processing). Thus, the TMS results show that Broca's region is causally involved in artificial syntax processing (Figure 6).

\section{A Theoretical Approach to Language Learning}

The final section of this article is devoted to a theoretical formulation of the language learning problem from a mechanistic neurobiological point of view and we discuss the logical possibility of combining the notion of innate language constraints with, for example, the notion of domain-general learning mechanisms, and several other interesting possibilities. In this context we approach 


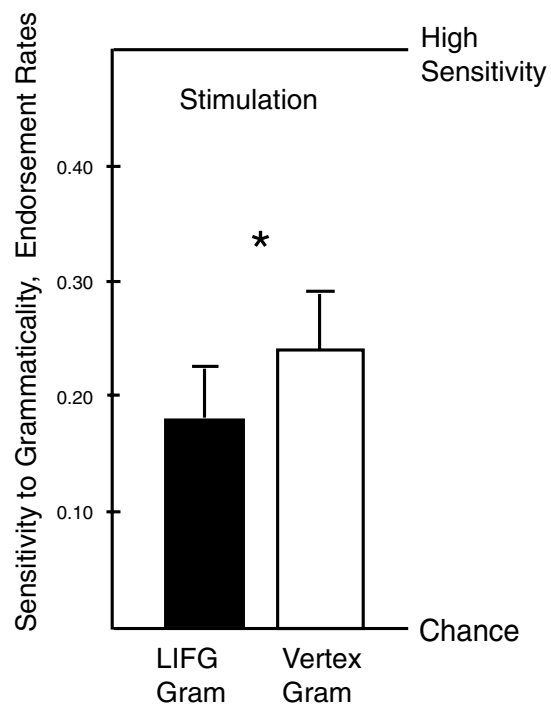

Figure 6 The difference in endorsement rates between grammatical and nongrammatical items in each classification test, with rTMS to left inferior frontal gyrus (LIFG) or vertex. The difference in endorsement rates between grammatical and nongrammatical items in the two classification test with stimulation is depicted. rTMS to Broca's region (BA 44/45) lead to significantly impaired classification performance, compared to control stimulation at vertex. The zero level on the $y$-axis = chance performance. "Gram" $=$ Grammaticality.

the brain as an adaptive information processing device - that is, a physical system that is viewed as a computational system with the capacity to change its way of processing information. This entails that a subclass of its states is viewed as representations and that transitions between states can be understood as a process implementing operations on the corresponding representational structures. Here, we use the concept information in a general sense-that is, equivalent with the notion of interpreted variability. In other words, for variability in some variable to be viewed as information presupposes a device or system that translates this variability into an (abstract) action of some sort (i.e., its interpretation or "processing role" in the system).

Here, we briefly outline some aspects of the information processing concept at a theoretical level. This will serve as background to the notion of language learning as adaptation of a dynamical system. We start by formalizing the classical Church-Turing architecture (Davis, Sigal, \& Weyuker, 1994; Turing, 1936a, 1936b; Wells, 2005) as a dynamical system and indicate that essentially the same concepts underlie nonclassical architectures (Petersson, 


\section{Adaptive Information Processing System}

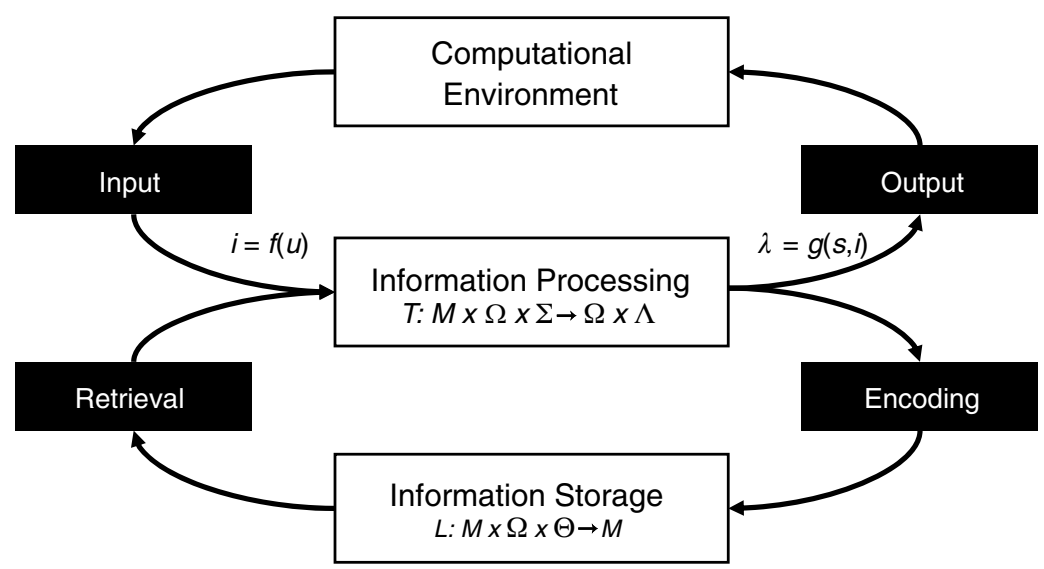

Figure 7 Adaptive information processing system (for formal details, see Petersson, 2005a).

2005a, 2008; Petersson, Ingvar, \& Reis, 2009; Siegelmann, 1999; Siegelmann \& Fishman, 1998). However, from a mechanistic neurobiological point of view, the nonclassical architectures - in particular, spiking recurrent neural network architectures - are in a sense closer in nature to real neurobiological systems.

To allow the reader to skip to the final section on formal learning theory, we summarize the theoretical outcome here: A developmental learning system is formalized as coupled dynamical systems, one for information processing that interacts with other dynamical subsystems for learning, memory, and development. Here, an adaptive dynamical system $C$ (Figure 7) is specified by the following: (a) its functional architecture - a specification of the structural organization of the system and the nature of its primitive processing units; (b) its representational dynamics - a specification of a state-space, $\Omega$, of state variables $s$ (e.g., membrane potentials) carrying information, and dynamical principles, $T$, governing state transitions (i.e., active processing of information); and, finally, (c) one (or several) learning dynamics - a specification of learning (adaptive) variables/parameters $m$ (e.g., synaptic parameters) for information storage (memory formation), and another set of dynamical principles $L$ (e.g., Hebbian learning) that govern the temporal evolution of the learning variables in a model space $M$. In short, $C=$ 〈functional architecture, representational dynamics, learning dynamics $>$ and the analysis below results in the following picture: Language acquisition is the result of an interaction between two sources 
of information: (a) innate prior structure, which is likely to be of a prelinguistic, non-language-specific type (i.e., phylogenetically predating the emergence of language) as well as language-specific to some extent; (b) the environment, both the linguistic and the extralinguistic, which can be viewed as interactive boundary conditions for the developing system. This is in its essentials similar to Chomsky's more recent formulations of language acquisition (Chomsky, 1980a, 1986) but holds more generally for any learning system.

The notions of representations and state transitions are formalized as a state-space $\Omega$ of internal states, and a transition mapping, $\mathrm{T}: \Omega \times \Sigma \rightarrow \Omega \times \Lambda$ (Figure 7), which govern how the information is being processed. Here, $\Sigma$ is the (transduced) input space and $\Lambda$ is the output space. In this picture, information processing (i.e., the state transitions) is nothing but input-driven trajectories in a suitable state-space $\Omega$ : At time step $t$, the system receives input $i(t)$ (in $\Sigma$ ), being in state $s(t)$ (in $\Omega$ ), and as a result of processing the joint information $[s(t), i(t)]$ (in $\Omega \times \Sigma)$, the system changes state to $s(t+\Delta t)$ and outputs $\lambda(t+$ $\Delta t$ ) (in $\Lambda$ ) according to the transition mapping $\mathrm{T}$ :

$$
[s(t+\Delta t), \lambda(t+\Delta t)]=T[s(t), i(t)]
$$

Equation 1 is the formal description of a generic discrete-time dynamical system that interfaces its (computational) environment through an input interface $i=f(u)$ (a transduction mechanism) and an output interface $\lambda=g(s, i)$. This represents a generic formulation of classical cognitive models viewed as realtime computational mechanisms. This framework assumes that information is coded by structured representations, data structures $(\sim[s, i])$, and that cognitive processing is accomplished by the execution of algorithmic operations (rules; $\sim \mathrm{T}$ in Figure 7) on compositionally structured representations (Newell \& Simon, 1976). Language modeling in theoretical linguistics and psycholinguistics represent examples in which the classical framework has served well. All common formal language models viewed as, for example, parsers or transducers can be described within the classical Church-Turing framework (Davis et al., 1994; Partee, ter Meulen, \& Wall, 1990). In this context, T would, for example, represent a parser, $s(t)$, the current parse, and $i(t)$, an incoming lexical item (for a concrete example, see Petersson et al., 2010).

In order to incorporate learning and development in this picture, the processing dynamics, $\mathrm{T}$, has to be complemented with a learning or developmental dynamics $\mathrm{L}: \mathrm{M} \times \Omega \times \Theta \rightarrow \mathrm{M}$ (Figure 7). For simplicity's sake, let $\mathrm{C}$ be the language system and let $\mathrm{T}$ be the parser associated with $\mathrm{C}$. Learning and/or development of the parsing capacity related to $\mathrm{C}$ means that $\mathrm{T}$ changes its 
processing characteristics over time. We can conceptualize the development of $\mathrm{C}$, or rather $\mathrm{T}$, as a trajectory in a model-space $\mathrm{M}=\{m \mid m$ can be instantiated by $C$, where a given $m$ would roughly correspond to an abstract state of the language system in a parameterized model of the language system. In other words, at any point in time, $t$, one can imagine $\mathrm{C}$ being in a given model-state $m(t)$. Note that state here refers to a model in model-space, currently instantiated by $\mathrm{C}$, rather than a cognitive state in the state-space of the processing system. If $\mathrm{C}$ incorporates an innately specified prior structure, we can capture this notion in four different ways: (a) by a structured initial state $m\left(t_{0}\right)$; (b) constraints on the model-space $\mathrm{M}$; (c) constraints on the representational state-space $\Omega$; or (d) domain specifications, incorporated in the learning/developmental dynamics L:M $\times \Omega \times \Theta \rightarrow$ M. Thus, as $\mathrm{C}$ develops (or acquires knowledge through learning), it traces out a trajectory in $\mathrm{M}$ determined by its adaptive dynamics $\mathrm{L}$ (Figure 7). If $\mathrm{C}$ and $\mathrm{L}$ are such that they (approximately) converge on a final model $m_{\mathrm{F}}$, this will characterize the end state of the developmental process reached after time $\Delta t_{\mathrm{F}}$ - that is, $m\left(t_{0}+\Delta t_{\mathrm{F}}\right) \approx m_{\mathrm{F}}$. In this picture, learning and development is viewed as two coupled dynamical systems, $\mathrm{T}$ and $\mathrm{L}$ (cf., Equations 1' and 2). Here, as $\mathrm{C}$ develops, it traces out a trajectory in $\mathrm{M}$ determined by its learning/developmental dynamics $\mathrm{L}$ according to

$$
m(t+\Delta t)=L(m(t), s(t), t)
$$

where the explicit dependence on time in L captures the idea of an innately specified developmental process (maturation) as well as a possible dependence on the previous developmental history of $\mathrm{C}$. Within this framework, $m$ determines the transition function $\mathrm{T}$ in the following sense: $\mathrm{T}$ can be viewed as parameterized by the current model $m$. In other words, $\mathrm{T}$ is not only a function of $[s, i]$ but is also a function of $m$ according to $\mathrm{T}=\mathrm{T}[m, s, i]$. More formally, the nonadaptive description $\mathrm{T}: \Omega \times \Sigma \rightarrow \Omega \times \Lambda$ is replaced by an adaptive formulation T:M $\times \Omega \times \Sigma \rightarrow \Omega \times \Lambda$, and Equation 1 needs to be modified according to

$$
[s(t+\Delta t), \lambda(t+\Delta t)]=T[m(t), s(t), i(t)] .
$$

Together, $\mathrm{T}$ and $\mathrm{L}$ form an adaptive dynamical system, and as already noted, this outline is in its essentials similar to Chomsky's more recent formulations of language acquisition (Chomsky, 1980a, 1986). 
Nonclassical architectures (Buonomano \& Maass, 2009; Gerstner \& Kistler, 2002; Maass \& Bishop, 2001; Maass, Joshi, \& Sontag, 2007; Rieke, Warland, de Ruyter van Steveninck, \& Bialek, 1999; Siegelmann, 1999; Siegelmann \& Fishman, 1998) require a different, more general formulation; for example, in terms of coupled multivariate stochastic differential/difference equation (e.g., Øksendal, 2000) with, for example, additive noise processes $\xi(t)$ and $\eta(t)$ :

$$
\begin{aligned}
& d s=T(s, m, i) d t+d \zeta(t), \\
& d m=\mathrm{L}(s, m, i) d t+d \eta(t) .
\end{aligned}
$$

However, the underlying conceptualization is identical with that of the classical architectures (Figure 8), although the nonclassical formulation-in particular, recurrent spiking neural network architectures - is more natural from a mechanistic neurobiological perspective. Here, the representational dynamics (information processing) corresponds to Equation 3 and the learning/developmental dynamics corresponds to Equation 4; a comparison with Equations 1' and 2 reveals that classical and nonclassical architectures share the same form. In this

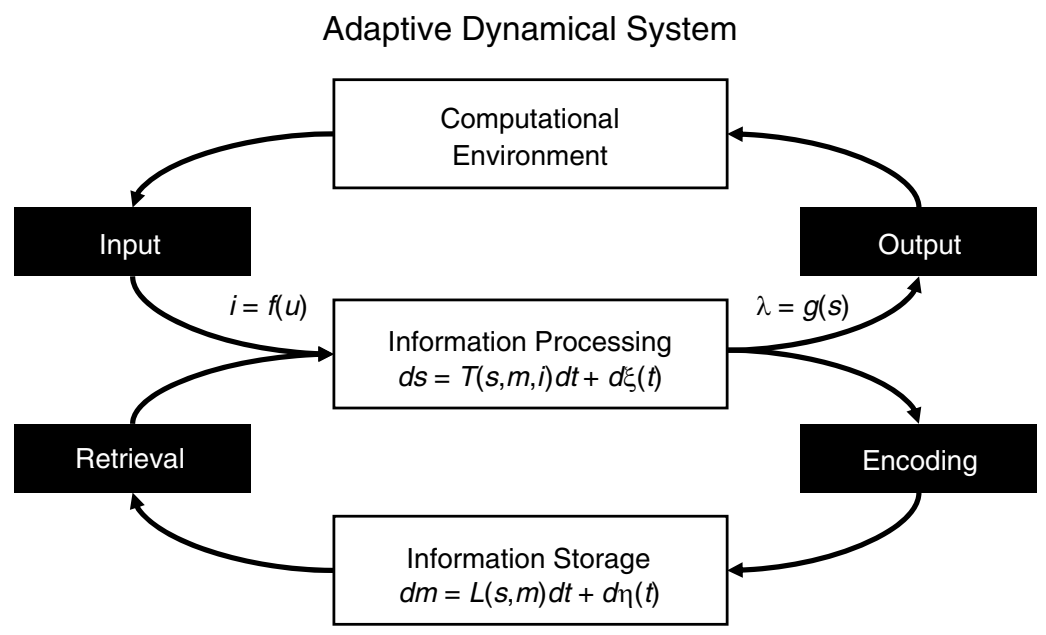

Figure 8 Adaptive dynamical system. Equations 3 and 4 specify how information processing interacts with the learning dynamics and can be directly related to, for example, the interaction between the perception-cognition-action and the encodingstorage-retrieval cycle, respectively. (For formal details, see Petersson, 2005a.) 
context, we note that it is uncontroversial that any physically realizable computational system is necessarily finite with respect to both its memory organization and processes information with finite precision (e.g., in the presence of noise). These constraints are examples of innate constraints that are nongenetic (for additional examples, see Cherniak, 2005).

\section{Implications for Formal Learning Theory}

With the formal framework in place, we now show that it is logically possible to combine the notion of innate language constraints with, for example, the notion of domain-general learning mechanisms, as well as other interesting possibilities within the framework of formal learning theory (e.g., Gold, 1967; Jain, Osherson, Royer, \& Sharma, 1999; Vapnik, 1998). In particular, we will elaborate on the meaning of Gold's Theorem and similar results (Gold, 1967; Jain et al., 1999; Vapnik, 1998). It is of interest to note that no infinite class of languages, including the class of regular languages, is, in general, learnable from positive examples alone without additional constraints on the specific learning paradigm (Gold, 1967; Jain et al., 1999). At first glance, this appears to exclude the possibility of learning artificial or natural grammars from positive examples alone; this seems to be the case also when statistical learning mechanisms are employed (Nowak, Komarova, \& Niyogi, 2002).

In Gold's (1967) classical learning framework, the learning system had to identify the target language exactly based on only positive examples (i.e., well-formed sequences). In addition, the environment provides an arbitrarily large number of examples to which the learning system has access. Issues related to computational complexity were ignored (see also Jain et al., 1999). However, already Gold noted that under suitable circumstances (i.e., with additional constraints on the learning paradigm), this (un)learnability result can be avoided. Such constraints may include, for example, the existence and effective use of explicit negative feedback, prior restrictions on the class of possible languages, or prior restrictions on the possible language experiences that can occur. Recent results in formal learning theory confirm Gold's suggestion that if the class of possible languages is restricted, then it is possible to learn infinite languages in infinite classes of formal languages from positive examples (Shinohara, 1994; Shinohara \& Arimura, 2000). It should be noted that these prior constraints on the class of possible (or accessible) languages are of a general type and not "language-specific" per se (e.g., restrictions on the maximal number of rules employed by the languages in the class). As noted by Scholz and Pullum (2002), there exists classes of formal languages rich enough to 
encompass the "string-sets" of human languages and at the same time identifiable from a finite sequence of positive examples. Clearly, the acquisition task becomes potentially more tractable if there is additional structure in the input or if only "probable approximate" identification is required. It has also been suggested that the acquisition of infinite classes of languages may be possible given reasonable probabilistic properties of the language environment and the initial language experience of children. Furthermore, negative evidence might be available based on expectations without explicit corrections (Rohde \& Plaut, 1999). We note two additional issues: (a) the problem of assuming that the class of possible languages is infinite and (b) the nature of the innate constraints. In the context of neural systems, which are physical devices, the notion of an infinite representational capacity that potentially supports any of an infinite number of possible languages is as problematic as the assumptions of infinite memory capacity or infinite processing precision (see discussion in Petersson, 2005b; Petersson et al., 2010). This insight, that the human brain has finite representational capacity, is originally Turing's (1936a, 1936b). Thus, in all likelihood, Gold's assumption of an infinite set of possible languages is misconceived if interpreted as a statement about possible end states of the neurobiological language faculty. If the set of possible human languages is finite, then this fact will make the acquisition problem easier than envisioned in Gold's Theorem. One may speculate therefore that the human brain can instantiate only a finite number of possible languages, which is another example of an innate but nongenetic constraint. Moreover, even if one accepts the conclusion of Gold's Theorem - the necessity of innate constraints - this conclusion does not specify the nature of these constraints.

Two facts about language learning seem indisputable: (a) Only humans and no other species acquire language, and thus it seems clear that there must be some biological element that accounts for this unique ability; (b) it is also clear that no matter how much of a head start the learner gains through innate constraints, language is learned. It is a trivial insight that built-in structure need not and is not learned and that structure that is available in experience and is acquired need not be built in (Yang, 2004). Thus, both innate endowment and learning contribute to language acquisition, the result of which is an extremely sophisticated body of linguistic knowledge (Yang, 2004). In fact, based on the formal outline in the previous section, it is clear that unless restrictions are placed on the model-space M and the learning mechanism L, "learning" would simply reduce to storing experience.

Much of the current discussion concerning the nature of innate constraints is focused on whether these are linguistically specific (Chomsky, 1986, 2005; 
however, see Chomsky, 2007) or not (e.g., Christiansen \& Chater, 2008; Hornstein, 2009). We think this is an empirical issue-what is clear is that no interesting, complex form of learning is possible without constraints (Jain et al., 1999; Vapnik, 1998). However, Yang (2004) cited an interesting insight by Fodor (2001, pp. 107-108), "Chomsky can with perfect coherence claim that innate, domain specific [constraints] mediate language acquisition, while remaining entirely agnostic about the domain specificity of language acquisition mechanisms." What can this possibly mean? Looking back at the previous section, it is a statement about the domain scope of the learning/developmental dynamics L, which might be domain-general in form, but in the context of language acquisition, it operates on a model-space $M$ that is restricted by innate, language-specific constraints. In fact, in order to rule out innate, languagespecific constraints completely, it is necessary to establish that none of the following candidates carry such constraints: (a) the initial state $m\left(t_{0}\right)$; (b) the model-space M; (c) the learning/developmental dynamics L; (d) the representational state-space $\Omega$; or (e) the representational dynamics $T-$ a difficult, but important, empirical task for future research.

\section{Conclusions}

A growing body of empirical evidence suggests that the mechanisms involved in artificial language learning and structured sequence processing are shared with natural language acquisition and natural language processing. This includes evidence from functional neuroimaging studies using MRI, EEG, and TMS. Furthermore, behavioral results also suggest that artificial language learning/processing is relevant to natural language learning/processing. We argued that the representational capacity of the human brain is finite and thus can only instantiate a finite number of possible languages - an example of innate but nongenetic constraint. Finally, by theoretically analyzing a formal learning model, we highlighted Fodor's insight that it is logically possible to combine innate, domain-specific constraints with domain-general learning mechanisms.

\section{References}

Andoh, J., Artiges, E., Pallier, C., Riviere, D., Mangin, J. F., Cachia, A., et al. (2006).

Modulation of language areas with functional MR image-guided magnetic stimulation. NeuroImage, 29, 629-627.

Aronoff, M., Meir, I., Padden, C., \& Sandler, W. (2008). The roots of linguistic organization in a new language. Interaction Studies, 9, 133-153. 
Aslin, R. N., Saffran, J. R., \& Newport, E. L. (1998). Computation of conditional probability statistics by 8-month-old infants. Psychological Science, 9, 321-324.

Bahlmann, J., Schubotz, R. I., \& Friederici, A. D. (2008). Hierarchical artificial grammar processing engages Broca's area. NeuroImage, 42, 525-534.

Bookheimer, S. (2002). Functional MRI of language: New approaches to understanding the cortical organization of semantic processing. Annual Review of Neuroscience, 25, 151-188.

Boroojerdi, B., Grafman, J., \& Cohen, L. (2001). Enhancing analogic reasoning with rTMS over the left prefrontal cortex. Neurology, 56, 526-528.

Braine, M. D. S. (1987). What is learned in acquiring word classes: A step toward an acquisition theory. In B. MacWhinney (Ed.), Mechanisms of language acquisition (pp. 65-87). Philadelphia: Erlbaum.

Buonomano, D. V., \& Maass, W. (2009). State-dependent computations: Spatiotemporal processing in cortical networks. Nature Reviews Neuroscience, 10, 113-125.

Chater, N., \& Christiansen, M. H. (2007). Two views of simplicity in linguistic theory: Which connects better with cognitive science? Trends in Cognitive Sciences, 11, 324-326.

Chater, N., \& Christiansen, M. H. (2009). Language acquisition meets language evolution. Cognitive Science, 7, 1131-1157.

Chater, N., Reali, F., \& Christiansen, M. H. (2009). Restrictions on biological adaptation in language evolution. Proceedings of the National Academy of Sciences, United States of America, 106, 1015-1020.

Cherniak, C. (2005). Innateness and brain-wiring optimization: Non-genomic nativism. In A. Zilhão (Ed.), Cognition, evolution, and rationality: A cognitive science for the twenty-first century (pp.103-112). London: Routledge.

Chomsky, N. (1965). Aspects of the theory of syntax. Cambridge, MA: MIT Press.

Chomsky, N. (1980a). On the biological basis of language capacities. In N. Chomsky (Ed.), Rules and representations (pp. 185-216). Oxford: Blackwell.

Chomsky, N. (1980b). Rules and representations. Behavioral and Brain Sciences, 3, $1-61$.

Chomsky, N. (1986). Knowledge of language. New York: Praeger.

Chomsky, N. (2005). Three factors in language design. Linguistic Inquiry, 36, 1-22.

Chomsky, N. (2007). Of mind and language. Biolinguistics, 1, 9-27.

Chomsky, N., \& Lasnik, H. (1995). The theory of principles and parameters. In N. Chomsky (Ed.), The minimalist program (pp. 1-13). Cambridge, MA: MIT Press.

Christiansen, M. H., \& Chater, N. (1999). Toward a connectionist model of recursion in human linguistic performance. Cognitive Science, 23, 157-205.

Christiansen, M. H., \& Chater, N. (2008). Language as shaped by the brain. Behavioral and Brain Sciences, 31, 489-558.

Christiansen, M. H., Kelly, L., Shillcock, R., \& Greenfield, K. (2010). Impaired artificial grammar learning in agrammatism Cognition, 116, 382-393. 
Christiansen, M. H., \& Kirby, S. (2003). Language evolution: Consensus and controversies. Trends in Cognitive Sciences, 7, 300-307.

Davis, M. D., Sigal, R., \& Weyuker, E. J. (1994). Computability, complexity, and languages: Fundamentals of theoretical computer science. San Diego, CA: Academic Press.

de Vries, M. H., Monaghan, P., Knecht, S., \& Zwitserlood, P. (2008). Syntactic structure and artificial grammar learning: The learnability of embedded hierarchical structures. Cognition, 107, 763-774.

de Vries, M. H., Monaghan, P., Knecht, S., \& Zwitserlood, P. (2010). Learning recursive structure in artificial languages: A different role for vowels and consonants. Manuscript submitted for publication.

Devlin, J. T., Matthews, P. M., \& Rushworth, M. F. (2003). Semantic processing in the left inferior prefrontal cortex: A combined functional magnetic resonance imaging and transcranial magnetic stimulation study. Journal of Cognitive Neuroscience, 15, 71-84.

Epstein, C. M., Meador, K. J., Loring, D. W., Wright, R. J., Weissman, J. D., Sheppard, S., et al. (1999). Localization and characterization of speech arrest during transcranial magnetic stimulation. Clinical Neurophysiology, 110, 1073-1079.

Evans, J. L., Saffran, J. R., \& Robe-Torres, K. (2009). Statistical learning in children with specific language impairment. Journal of Speech, Language, and Hearing Research, 52, 321-335.

Evans, N., \& Levinson, S. C. (2009). The myth of language universals: Language diversity and its importance for cognitive science. Behavioral and Brain Sciences, 32, 429-492.

Fitch, W. T., \& Hauser, M. D. (2004). Computational constraints on syntactic processing in a nonhuman primate. Science, 303, 377-380.

Flitman, S., Grafman, J., Wassermann, E., Cooper, V., O'Grady, J., Pascual-Leone, A., \& Hallett, M. (1998). Linguistic processing during repetitive transcranial magnetic stimulation. Neurology, 50, 175-181.

Fodor, J. A. (2001). Doing without what's within: Fiona Cowie's criticism of nativism. Mind, 110, 99-148.

Folia, V., Forkstam, C., Hagoort, P., \& Petersson, K. M. (2009). Language comprehension: The interplay between form and content. In N. A. Taatgen \& H. van Rijn (Eds.), Proceedings of the 31st annual conference of the Cognitive Science Society, 2009 (pp. 1686-1691). Austin, TX: Cognitive Science Society.

Folia, V., Uddén, J., Forkstam, C., Ingvar, M., Hagoort, P., \& Petersson, K. M. (2008). Implicit learning and dyslexia. Annals of the New York Academy of Sciences, 1145, 132-150.

Forkstam, C., Elwér, Å., Ingvar, M., \& Petersson, K. M. (2008). Instruction effects in implicit artificial grammar learning: A preference for grammaticality. Brain Research, 1221, 80-92. 
Forkstam, C., Hagoort, P., Fernandez, G., Ingvar, M., \& Petersson, K. M. (2006). Neural correlates of artificial syntactic structure classification. NeuroImage, 32, 956-967.

Forkstam, C., Jansson, A., Ingvar, M., \& Petersson, K. M. (2009). Modality transfer of acquired structural regularities: A preference for an acoustic route. In N. A. Taatgen \& H. van Rijn (Eds.), Proceedings of the 31st annual conference of the Cognitive Science Society, 2009 (pp. 1977-1982). Austin, TX: Cognitive Science Society.

Forkstam, C., \& Petersson, K. M. (2005). Towards an explicit account of implicit learning. Current Opinion in Neurology, 18, 435-441.

Friederici, A. D., Bahlmann, J., Heim, S., Schubotz, R. I., \& Anwander, A. (2006). The brain differentiates human and non-human grammars: Functional localization and structural connectivity. Proceedings of the National Academy of Sciences, United States of America, 103, 2458-2463.

Friederici, A. D., Fiebach, C. J., Schlesewsky, M., Bornkessel, I. D., \& von Cramon, D. Y. (2006). Processing linguistic complexity and grammaticality in the left frontal cortex. Cerebral Cortex, 16, 1709-1717.

Friederici, A. D., Steinhauer, K., \& Pfeifer, E. (2002). Brain signatures of artificial language processing: Evidence challenging the critical period hypothesis. Proceedings of the National Academy of Sciences, United States of America, 99, 529-534.

Gentner, T. Q., Fenn, K. M., Margoliash, D., \& Nusbaum, H. C. (2006). Recursive syntactic pattern learning by songbirds. Nature, 440, 1204-1207.

Gerken, L. (2006). Decisions, decisions: Infant language learning when multiple generalizations are possible. Cognition, 98, B67-B74.

Gerken, L., Wilson, R., \& Lewis, W. (2005). Infants can use distributional cues to form syntactic categories. Journal of Child Language, 32, 249-268.

Gerstner, W., \& Kistler, W. (2002). Spiking neuron models: Single neurons, populations, plasticity. Cambridge: Cambridge University Press.

Gold, E. M. (1967). Language identification in the limit. Information and Control, 10, 447-474.

Gómez, R. L., Bootzin, R. R., \& Nadel, L. (2006). Naps promote abstraction in language-learning infants. Psychological Science, 17, 670-674.

Gómez, R. L., \& Gerken, L. (1999). Artificial grammar learning by 1-year-olds leads to specific and abstract knowledge. Cognition, 70, 109-135.

Gómez, R. L., \& Gerken, L. (2000). Infant artificial language learning and language acquisition. Trends in Cognitive Sciences, 4, 178-186.

Gómez, R. L., \& Maye, J. (2005). The developmental trajectory of nonadjacent dependency learning. Infancy, 7, 183-206.

Gough, P. M., Nobre, A. C., \& Devlin, J. T. (2005). Dissociating linguistic processes in the left inferior frontal cortex with transcranial magnetic stimulation. Journal of Neuroscience, 25, 8010-8016. 
Grodzinsky, Y., \& Santi, A. (2008). The battle for Broca's region. Trends in Cognitive Sciences, 12, 474-480.

Hagoort, P. (2005). On Broca, brain, and binding: A new framework. Trends in Cognitive Sciences, 9, 416-423.

Hauser, M. D., Chomsky, N., \& Fitch, W. T. (2002). The faculty of language: What is it, who has it, and how did it evolve? Science, 298, 1569-1579.

Hauser, M. D., Weiss, D. J., \& Marcus, G. F. (2002). Rule learning by cotton-top tamarins. Cognition, 86, B15-B22.

Hockett, C. F. (1963). The problem of universals in language. In J. H. Greenberg (Ed.), Universals of language (pp. 1-29). Cambridge, MA: MIT Press.

Hockett, C. F. (1987). Refurbishing our foundations: Elementary linguistics from an advanced point of view. Philadelphia: Benjamins.

Hoen, M., \& Dominey, P. F. (2000). ERP analysis of cognitive sequencing: A left anterior negativity related to structural transformation processing. NeuroReport, 11, 3187-3191.

Hoen, M., Golembiowski, M., Guyot, E., Deprez, V., Caplan, D., \& Dominey, P. F. (2003). Training with cognitive sequences improves syntactic comprehension in agrammatic aphasics. NeuroReport, 14, 495-499.

Hornstein, N. (2009). A theory of syntax: Minimal operations and universal grammar. Cambridge: Cambridge University Press.

Hsu, H.-J., Christiansen, M. H., Tomblin, J. B., Zhang, X., \& Gómez, R. L. (2006, June). Statistical learning of nonadjacent dependencies in adolescents with and without language impairment. Poster presented at the 2006 Symposium on Research in Child Language Disorders, Madison, WI.

Jackendoff, R. (2002). Foundations of language: Brain, meaning, grammar, evolution. Oxford: Oxford University Press.

Jain, S., Osherson, D., Royer, J. S., \& Sharma, A. (1999). Systems that learn. Cambridge, MA: MIT Press.

Joshi, A. K., \& Schabes, Y. (1997). Tree-adjoining grammars. In G. Rosenberg \& A. Salomaa (Eds.), Handbook of formal languages (pp. 69-123). Berlin: Springer.

Kang, B. (2008). Acquisition of language particular properties under impoverished input. Language Acquisition, 15, 123-125.

Kirkham, N. Z., Slemmer, J. A., \& Johnson, S. P. (2002). Visual statistical learning in infancy: Evidence for a domain general learning mechanism. Cognition, 83, B35-B42.

Kirschen, M. P., Davis-Ratner, M. S., Jerde, T. E., Schraedley-Desmond, P., \& Desmond, J. E. (2006). Enhancement of phonological memory following transcranial magnetic stimulation (TMS). Behavioural Neurology, 17, 187-194.

Knowlton, B. J., \& Squire, L. R. (1996). Artificial grammar learning depends on implicit acquisition of both abstract and exemplar-specific information. Journal of Experimental Psychology: Learning, Memory, and Cognition, 22, 169-181. 
Lidz, J., Waxman, S., \& Freedman, J. (2003). What infants know about syntax but couldn't have learned: Experimental evidence for syntactic structure at 18 months. Cognition, 89, B65-B73.

Maass, W., \& Bishop, C. M. (Eds.). (2001). Pulsed neural networks. Cambridge, MA: MIT Press.

Maass, W., Joshi, P., \& Sontag, E. (2007). Computational aspects of feedback in neural circuits. PLoS Computational Biology, 3(1), e165. doi:110.1371/journal.pcbi. 0020165.

MacWhinney, B. (2000). The CHILDES Project: Tools for analyzing talk: Vol. II. The database (3rd ed.). Mahwah, NJ: Erlbaum.

Makuuchi, M., Bahlmann, J., Anwander, A., \& Friederici, A. D. (2009). Segregating the core computational faculty of human language from working memory.

Proceedings of the National Academy of Sciences, United States of America, 106, 8362-8367.

Marcus, G. F., Johnson, S. P., Fernandes, K. J., \& Slemmer, J. A. (2004, November). Rules, statistics and domain-specificity: Evidence from prelinguistic infants. Paper presented at the 29th Annual Meeting of the Boston University Conference on Language Development, Boston, MA.

Marcus, G. F., Vijayan, S., Bandi Rao, S., \& Vishton, P. M. (1999). Rule learning by seven-month-old infants. Science, 283, 77-80.

Meulemans, T., \& Van der Linden, M. (1997). Associative chunk strength in artificial grammar learning. Journal of Experimental Psychology: Learning, Memory, and Cognition, 23, 1007-1028.

Misyak, J. B., Christiansen, M. H., \& Tomblin, J. B. (2009). Statistical learning of nonadjacencies predicts on-line processing of long-distance dependencies in natural language. In N. A. Taatgen \& H. van Rijn (Eds.), Proceedings of the 31st annual conference of the Cognitive Science Society, 2009 (pp. 177-182). Austin, TX: Cognitive Science Society.

Mottaghy, F. M., Sparing, R., \& Töpper, R. (2006). Enhancing picture naming with transcranial magnetic stimulation. Behavioural Neurology, 17, 177-186.

Newell, A., \& Simon, H. (1976). Computer science as empirical inquiry: Symbols and search. Communications of the Association for Computing Machinery, 19, 111-126.

Newport, E. L., \& Aslin, R. N. (2004). Learning at a distance: I. Statistical learning of non-adjacent dependencies. Cognitive Psychology, 48, 127-162.

Newport, E. L., Hauser, M. D., Spaepen, G., \& Aslin, R. N. (2004). Learning at a distance: II. Statistical learning of non-adjacent dependencies in a non-human primate. Cognitive Psychology, 49, 85-117.

Nieuwenhuis, I. L. C., Folia, V., Forkstam, C., Jensen, O., \& Petersson, K. M. (2010). Grammar learning requires sleep. Manuscript submitted for publication.

Nowak, M. A., Komarova, N. L., \& Niyogi, P. (2002). Computational and evolutionary aspects of language. Nature, 417, 611-617. 
O'Donnell, T. J., Hauser, M. D., \& Fitch, W. T. (2005). Using mathematical models of language experimentally. Trends in Cognitive Sciences, 9, 284-289.

Øksendal, B. (2000). Stochastic differential equations: An introduction with applications (5th ed.). Berlin: Springer.

Partee, B. H., ter Meulen, A., \& Wall, R. E. (1990). Mathematical methods in linguistics. Dordrecht: Kluwer Academic.

Pelucchi, B., Hay, J. F., \& Saffran, J. R. (2009). Statistical learning in a natural language by 8-month-old infants. Child Development, 80, 674-685.

Perruchet, P., \& Rey, A. (2005). Does the mastery of center-embedded linguistic structures distinguish humans from nonhuman primates? Psychonomic Bulletin and Review, 12, 307-313.

Petersson, K. M. (2005a). Learning and memory in the human brain. Stockholm: Karolinska University Press.

Petersson, K. M. (2005b). On the relevance of the neurobiological analogue of the finite-state architecture. Neurocomputing, 65-66, 825-832.

Petersson, K. M. (2008). On cognition, structured sequence processing and adaptive dynamical systems. Proceedings of the American Institute of Physics: Mathematical and Statistical Physics Subseries, 1060, 195-200.

Petersson, K. M., Folia, V., \& Hagoort, P. (in press). What artificial grammar learning reveals about the neurobiology of syntax. Brain and Language.

Petersson, K. M., Forkstam, C., \& Ingvar, M. (2004). Artificial syntactic violations activate Broca's region. Cognitive Science, 28, 383-407.

Petersson, K. M., Ingvar, M., \& Reis, A. (2009). Language and literacy from a cognitive neuroscience perspective. In D. R. Olson \& N. Torrance (Eds.), Cambridge handbook of literacy (pp. 152-182). Cambridge: Cambridge University Press.

Poletiek, F. H. (2002). Implicit learning of a recursive rule in an artificial grammar. Acta Psychologica, 111, 323-335.

Pullum, G. K., \& Scholz, B. C. (2002). Empirical assessment of stimulus poverty arguments. Linguistic Review, 19, 9-50.

Reali, F., \& Christiansen, M. H. (2009). Sequential learning and the interaction between biological and linguistic adaptation in language evolution. Interaction Studies, 10, 5-30.

Reber, A. S. (1967). Implicit learning of artificial grammars. Journal of Verbal Learning and Verbal Behavior, 6, 855-863.

Richardson, J., Harris, L., Plante, E., \& Gerken, L. (2006). Subcategory learning in normal and language learning-disabled adults: How much information do they need? Journal of Speech, Language, and Hearing Research, 49, 1257-1266.

Rieke, F., Warland, D., de Ruyter van Steveninck, R., \& Bialek, W. (1999). Spikes: Exploring the neural code. Cambridge, MA: MIT Press.

Rohde, D. L. T., \& Plaut, D. C. (1999). Language acquisition in the absence of explicit negative evidence: How important is starting small? Cognition, 72, 67-109. 
Saffran, J. R., Aslin, R. N., \& Newport, E. L. (1996). Statistical learning by 8-month-old infants. Science, 274, 1926-1928.

Saffran, J. R., Hauser, M. D., Seibel, R. L., Kapfhamer, J., Tsao, F., \& Cushman, F. (2008). Grammatical pattern learning by human infants and cotton-top tamarin monkeys. Cognition, 107, 479-500.

Saffran, J. R., Johnson, E. K., Aslin, R. N., \& Newport, E. L. (1999). Statistical learning of tone sequences by human infants and adults. Cognition, 70, 27-52.

Saffran, J. R., Pollak, S. D., Seibel, R. L., \& Shkolnik, A. (2007). Dog is a dog is a dog: Infant rule learning is not specific to language. Cognition, 105, 669-680.

Saffran, J. R., Reeck, K., Niebuhr, A., \& Wilson, D. (2005). Changing the tune: The structure of the input affects infants' use of absolute and relative pitch.

Developmental Science, 8, 1-7.

Sandler, W., Meir, I., Padden, C., \& Aronoff, M. (2005). The emergence of grammar: Systematic structure in a new language. Proceedings of the National Academy of Sciences, United States of America, 102, 2661-2665.

Santelmann, L. M., \& Jusczyk, P. W. (1998). Sensitivity to discontinuous dependencies in language learners: Evidence for limitations in processing space. Cognition, 69, $105-134$.

Santi, A., \& Grodzinsky, Y. (2007a). Taxing working memory with syntax: Bihemispheric modulation. Human Brain Mapping, 28, 1089-1097.

Santi, A., \& Grodzinsky, Y. (2007b). Working memory and syntax interact in Broca's area. NeuroImage, 37, 8-17.

Scholz, B. C., \& Pullum, G. K. (2002). Searching for arguments to support linguistic nativism. Linguistic Review, 19, 185-223.

Seger, C. A. (1994). Implicit learning. Psychological Bulletin, 115, 163-196.

Senghas, A., Kita, S., \& Özyürek, A. (2004). Children creating core properties of language: Evidence from an emerging sign language in Nicaragua. Science, 305, 1779-1782.

Shapiro, K. A., Pascual-Leone, A., Mottaghy, F. M., Gangitano, M., \& Caramazza, A. (2001). Grammatical distinctions in the left frontal cortex. Journal of Cognitive Neuroscience, 13, 713-720.

Shinohara, T. (1994). Rich classes inferable from positive data: Length-bounded elementary formal systems. Information and Computation, 108, 175-186.

Shinohara, T., \& Arimura, H. (2000). Inductive inference of unbound unions of pattern languages from positive data. Theoretical Computer Science, 241, 191-209.

Siegelmann, H. T. (1999). Neural networks and analog computation: Beyond the Turing limit. Basel: Birkhäuser.

Siegelmann, H. T., \& Fishman, S. (1998). Analog computation with dynamical systems. Physica D, 120, 214-235.

Stadler, M. A., \& Frensch, P. A. (Eds.). (1998). Handbook of implicit learning. London: Sage. 
Töpper, R., Mottaghy, F. M., Brügmann, M., Noth, J., \& Huber, W. (1998). Facilitation of picture naming by focal transcranial magnetic stimulation of Wernicke's area. Experimental Brain Research, 121, 371-378.

Turing, A. (1936a). On computable numbers with an application to the Entscheidungs problem (part 1). Proceedings of the London Mathematical Society, 42(3), 230-240.

Turing, A. (1936b). On computable numbers with an application to the Entscheidungs problem (part 2). Proceedings of the London Mathematical Society, 42(4), 241-265.

Uddén, J., Araujo, S., Forkstam, C., Ingvar, M., Hagoort, P., \& Petersson, K. M. (2009). A matter of time: Implicit acquisition of recursive sequence structures. In N. A. Taatgen \& H. van Rijn (Eds.), Proceedings of the 31st annual conference of the Cognitive Science Society, 2009 (pp. 2444-2449). Austin, TX: Cognitive Science Society.

Uddén, J., Ingvar, M., Hagoort, P., \& Petersson, K. M. (2010a). Broca 's region: A causal role in implicit processing of grammars with adjacent and non-adjacent dependencies. Manuscript submitted for publication.

Uddén, J., Ingvar, M., Hagoort, P., \& Petersson, K. M. (2010b). Implicit acquisition of grammars with crossed and nested non-adjacent dependencies: Investigating the push-down stack model. Manuscript submitted for publication.

Uddén, J., Folia, V., Forkstam, C., Ingvar, M., Fernandez, G., Overeem, S., et al. (2008). The inferior frontal cortex in artificial syntax processing: An rTMS study. Brain Research, 1224, 69-78.

Vapnik, V. (1998). Statistical learning theory. New York: Wiley.

Vosse, T., \& Kempen, G. (2000). Syntactic structure assembly in human parsing: A computational model based on competitive inhibition and a lexicalist grammar. Cognition, 75, 105-143.

Wells, A. (2005). Rethinking cognitive computation: Turing and the science of the mind. Hampshire, UK: Palgrave Macmillan.

Yang, C. D. (2004). Universal grammar, statistics or both? Trends in Cognitive Sciences, 8, 451-456.

Zajonc, R. B. (1968). Attitudinal effects of mere exposure. Journal of Personality and Social Psychology Monograph Supplement, 9(2, Part 2), 1-27. 